\section{Strength in Silk is Driven by Critically Sized Nanocrystals}

Silk has been a prized material for thousands of years, partially due to its high strength. This strength arises from protein nanocrystals folded into $\beta$-sheets, which act as a reinforcing phase within the silk fiber (see Figure 1). As described in the April issue of Nature Materials (DOI: 10.1038/nmat2704; p. 359), M.J. Buehler and co-researchers at the Massachusetts Institute of Technology used molecular simulations to identify $\sim 3 \mathrm{~nm}$ as a critical dimension of nanocrystals, which directly affects the elastic modulus, strength, and toughness of silk fibers.

Buehler and graduate student S. Keten, postdoctoral associate $\mathrm{Z}$. $\mathrm{Xu}$, and undergraduate student B. Ihle used the crystalline protein sequence expressed by the mulberry silkworm, Bombyx mori, to simulate nanocrystals ranging in size from approximately $2 \mathrm{~nm}$ to $6.8 \mathrm{~nm}$, in a series of molecular level computational experiments to probe the effect of crystal size on their mechanical properties. The results show that nanocrystals below $\sim 3 \mathrm{~nm}$ are much stronger and tougher than those at larger dimensions. The researchers explain this observation by the increased significance of shear, which dominates the mechanics of nanocrystals below this size and that allows all hydrogen bonds to work concertedly. This results in a significant buildup of mechanical strength. Taking into account the effect of shear, the calculated values for elastic $(E=22.6 \mathrm{GPa})$ shear $(G=4.6 \mathrm{GPa})$ moduli correlated well to experimental values. Simulated pull-out tests, used to identify the nanocrystals' failure mechanisms, demonstrated that nanocrystals below a critical size of $\sim 3 \mathrm{~nm}$ exhibit a highly dissipative slip-stick behavior. This slip-stick motion, enabled by the weak nature of hydrogen bonds, dissipates a significant amount of energy
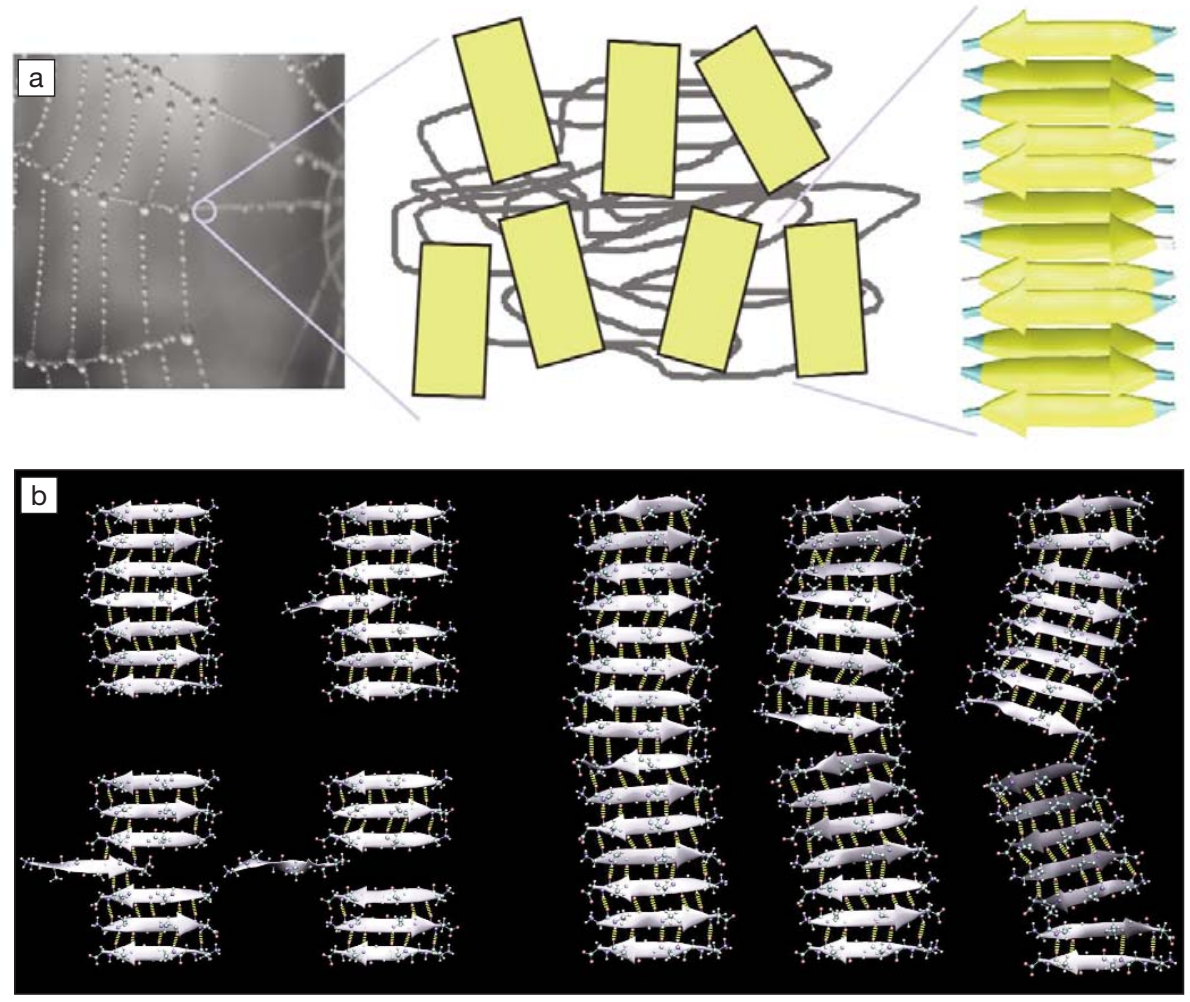

Figure 1. (a) Hierarchical structure of silk, which contains $\beta$-sheet nanocrystals. Hydrogen bonding occurs between the sheets, designated as arrows. (b) Breaking mechanisms of silk nanocrystals. The left part of the image shows a small crystal, which fails gracefully as a strand is being pulled out. The larger crystal on the right fails catastrophically as a crack forms at the left part. Figure courtesy M. Buehler (MIT). Reproduced with permission from Nature Materials 9 (4) (2010) 360; DOI: 10.1038/nmat2704. (C2010 Nature Publishing Group.

and allows nanocrystals below $\sim 3 \mathrm{~nm}$ to achieve a much greater strength, toughness, and resilience.

This work shows that while hydrogen bonds are typically weak, confinement of these bonds to nanometer-sized domains can impart considerable mechanical strength, resolving a long-standing question that has puzzled materials researchers for decades. In the case of silk, this strength can exceed that of steel. The results from this study may lead to the design of new, synthetic materials such as ultrahigh strength, light-weight composites, and may help to overcome the limitations of current engineered materials to achieve greater strength at greater toughness, simultaneously.

SCOTT COOPER

\section{Gold Nanoparticle Arrays Display Plasmon-Induced Electrical Conduction}

Fabrication at the nanoscale is fundamental to both molecular electronics and plasmonics, where optical interactions with nanoparticles induce surface plasmons (localized, collective oscillations of conduction electrons on the nanoparticle surface) creating locally intense electromagnetic fields. Applications that utilize surface plasmons include surfaceenhanced Raman spectroscopy, targeted tumor treatment, and molecular recognition labeling. In addition, progress made in several critical aspects of molecular electronics has resulted in useful devices. However, yet to be observed is the interaction of optically induced plasmons resulting in charge migration through single molecules. Recently, though, D.A. Bonnell of the University of Pennsylvania, P. Banerjee of the University of Maryland, M.J. Therien of Duke University, and their colleagues have demonstrated the ability of surface plasmons to alter the electrical properties of a molecular junction by coupling gold nanoparticle arrays with highly conjugated, chromophoric wires.

As reported in the February 23 issue of
ACS Nano (DOI: 10.1021/nn901148m; p. 1019), Bonnell and co-researchers chose a device platform consisting of an array of $\mathrm{Au}$ nanoparticles on an insulating glass substrate. These well-studied systems exhibit conduction that depends on nanoparticle spacing and occurs according to a percolation mechanism. The researchers controlled nanoparticle spacing by coating the Au nanoparticles with $\alpha, \omega$-dithiolterminated meso-ethyne-bridged porphyrin supermolecules (dithiol-PZn 3 ), which serve as 4.6-nm bridges between $\mathrm{Au}$ nanoparticles (see Figure 1). In addition, dithiol-PZn $\mathrm{n}_{3}$ bridges possess optoelec- 